\section{Is Allergic Sensitization to Siberian Hamster Preventable in High-Risk Individuals Who Are Already Sensitized or Exposed to Furry Animals?}

Liccardi $G^{1,2}$, Salzillo A ${ }^{1}$, Calzetta L ${ }^{3}$, Piccolo A ${ }^{1}$, Puxeddu E $E^{2,3}$, Rogliani $\mathrm{P}^{2,3}$

${ }^{1}$ Department of Pulmonology, Haematology and Oncology, Division of Pneumology and Allergology, High Speciality "A.Cardarelli" Hospital, Naples, Italy

${ }^{2}$ Postgraduate School of Respiratory Medicine, Department of Systems Medicine, University of Rome "Tor Vergata", Rome, Italy ${ }^{3}$ Department of Systems Medicine, University of Rome "Tor Vergata", Rome, Italy

J Investig Allergol Clin Immunol 2016; Vol. 26(6): 403-405 doi: $10.18176 /$ jiaci.0100

Key words: Allergic rhinitis. Allergic sensitization. Bronchial asthma. Component-resolved diagnosis. Hypersensitivity. Pet allergy. Siberian hamster.

Palabras clave: Rinitis alérgica. Sensibilización alérgica. Asma bronquial. Diagnóstico por components. Hipersensibilidad. Alergia a animales domésticos. Hámster siberiano.

\section{To the Editor:}

We read with interest the article from Torres et al [1] showing that exposure to Siberian hamster $(\mathrm{SH})$ in sensitized patients can elicit intense respiratory symptoms characterized by a relatively rapid onset after the initial exposure. The article contains a large and well-conducted study of allergy to $\mathrm{SH}$ on which we have nothing to comment. However, we would like to provide some suggestions on prevention of allergic sensitization to $\mathrm{SH}$ based on our clinical and scientific experience.

In recent years, hamsters have become increasingly popular as pets. For instance, in Japan, hamster ownership increased considerably in the 1990 s (to reach about $20 \%$ of all pets), as did the number of hamster owners who began to report respiratory symptoms [2]. The popularity of the $\mathrm{SH}$ is probably due to the fact that it is more easily cared for than other pets. It is also possible that, in certain circumstances, the SH may be considered an alternative pet for patients with potential or known allergic sensitization to dogs, cats, or other furry animals. This hypothesis seems to be confirmed by the finding that only 3 of the 21 patients included in the study of Torres et al [1] reported no previous contact with other pets. It is not clear whether the remaining 18 patients had been exposed (as indicated in the table) or had been exposed and were sensitized to other animals, as reported in the study.
Undoubtedly, the presence of a new furry pet at home can significantly increase the amount of allergens indoors. However, merely keeping an SH at home should not be considered a key risk factor for allergic sensitization in susceptible individuals $[3,4]$.

In the Naples area, only about $50 \%$ of atopic patients sensitized to common pets (dogs and cats) are directly exposed to these animals, whereas the other half are indirectly exposed (through contact with dog and cat owners) or not exposed [5]. If we consider allergic sensitization and modalities of exposure to other furry animals such as rabbits, hamsters, rats, horses, cows, and mice, the percentage of sensitized individuals directly exposed to these animals ranges between $0 \%$ and $33.3 \%$, whereas sensitized patients with indirect or no animal contact ranges from $66.7 \%$ to $100 \%$ [5]. Dog and cat allergens should be considered ubiquitous because they are found not only in the indoor environments where these animals are kept, but also in other private or public indoor areas where they have never been kept [4]. In developed countries, the ubiquity of pet allergens is responsible for persistent stimulation of airways, similar to that induced by dust mite and leading to an increased risk of allergic sensitization either directly or by cross-reactive mechanisms involving albumins and lipocalins [6]. In other words, a considerable number of patients included in the study of Torres et al [1] may have already been sensitized to hamster epithelial allergens (lipocalins), as described above. The introduction of an $\mathrm{SH}$ at home could have further increased allergic sensitization (to urine- and salivary glandderived allergens) and, thus, triggered respiratory symptoms. Moreover, it was not specified whether the 3 patients who denied previous (direct?) animal exposure had been indirectly exposed to dogs and cats and therefore potentially already sensitized.

Finally, using an in vivo model (skin prick test), we showed that exposure and allergic sensitization to common pets increases by about 14-fold the risk of developing sensitization to other furry animals, thus suggesting a possible predisposition to sensitization to multiple animal allergens (allergic phenotype?) [7]. We also recently confirmed these findings in vitro using a microarray technique (ImmunoCAP ISAC, Thermo Fisher Scientific). Our data suggest that allergic sensitization to common pets increases the risk of sensitization to horse and mouse allergens because of the presence of lipocalins [8].

Therefore, we investigated how to estimate the risk of sensitization after acquisition of a small pet such as the $\mathrm{SH}$ in patients who are already sensitized to dogs and cats and in those who have already removed common pets from their homes. These issues are particularly crucial if the pet-sensitized patients are children who wish to own a new pet (eg, hamsters) or to come into contact with larger animals in settings such as zoos, circuses, and farms. 


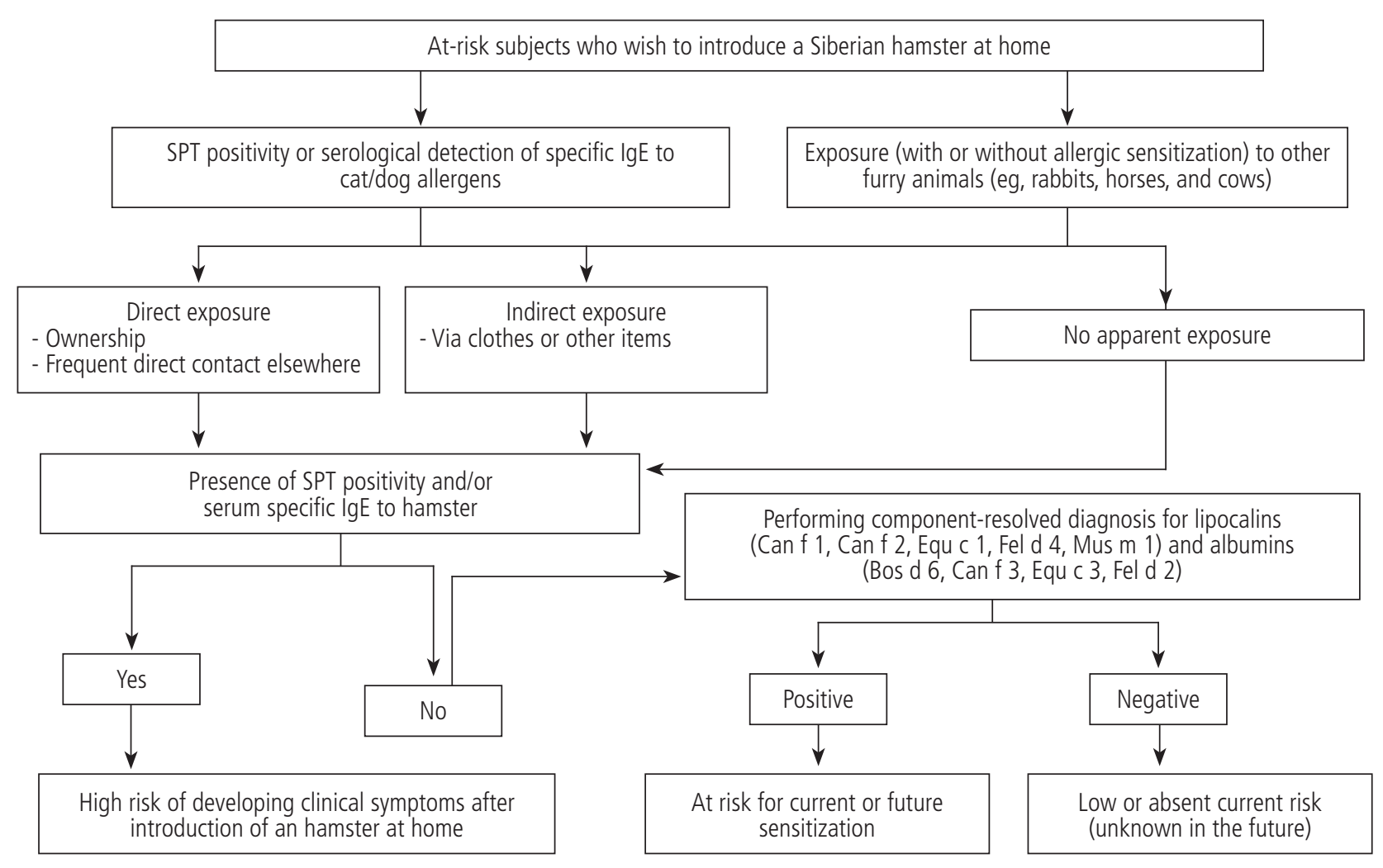

Figure. Flowchart for evaluating the risk of developing allergic sensitization to Siberian hamster in susceptible individuals who wish to own this pet. SPT indicates skin prick test.

In the Figure, we present a diagnostic flowchart for susceptible individuals who wish to own an $\mathrm{SH}$, although these criteria may also be useful for other small pets such as rabbits. In fact, rabbits can induce allergic sensitization by both epithelial and urinary allergens [9].

In conclusion, there is no doubt that the introduction of an $\mathrm{SH}$ at home can induce intense respiratory symptoms in sensitized individuals. It is important to highlight that allergic sensitization without direct animal exposure is a potential risk for patients, because they may often be unaware that severe respiratory symptoms can develop, even after occasional contact with an animal. Susceptible individuals should take this possibility into account before acquiring new pets or entering into contact with common and uncommon animals as part of their work or leisure activities. As a consequence, we believe that patients who are already sensitized and those who are directly exposed to furry animals should undergo accurate diagnostic evaluation before purchasing an $\mathrm{SH}$ as a pet.

Skin prick tests and/or evaluation of specific IgE to less common mammalian allergens should be highly recommended in these individuals in order to identify allergic sensitization and, consequently, prevent exposure. In this context, an evaluation of specific IgE using microarray techniques (component-resolved diagnosis) for lipocalins (Can $\mathrm{f} 1$, Can f 2, Equ c 1, Fel d 4, and Mus m 1) and albumins (Bos d 6, Can f 3, Equ c 3, and Fel d 2) could prove very useful when evaluating the possibility of cross-reactions between allergens from different animals [10].

\section{Funding}

The authors declare that no funding was received for the present study.

\section{Conflicts of Interest}

The authors declare that they have no conflicts of interest.

\section{References}

1. Torres JA, Sastre J, Vivanco F, Sanz Maroto A, de las Heras M, Pastor-Vargas C. Clinical characteristics of patients sensitized to Siberian hamster. J Investig Allergol Clin Immunol. 2016;26:113-5.

2. Suzuki K, Kayaba K, Tanuma T, Kitazawa J, Yanagewa $H$. Respiratory symptoms to hamster or other pets: a largesized population survey in Saitama Prefecture. J Epidemiol. 2005;15:9-14.

3. Liccardi G, Salzillo A, Cecchi L, D'Amato M, D'Amato G. Is cat keeping the main determinant of new-onset adulthood cat sensitization? J Allergy Clin Immunol. 2012;129:1689-90.

4. Liccardi G, Salzillo A, Calzetta L, Piccolo A, Menna G, Rogliani P. Can the presence of cat/dog at home be considered the only criterion of exposure to cat/dog allergens? A likely underestimated bias in clinical practice and in large epidemiological studies. Eur Ann Allergy Clin Immunol. 2016;48:61-4 
5. Liccardi G, Salzillo A, Piccolo A, Russo M, D'Amato G. Sensitization to furry animals in an urban atopic population living in Naples, Italy. Allergy. 2011;66:1500-1.

6. Liccardi G, Asero R, D'Amato M, D'Amato G. Role of sensitization to mammalian serum albumin in allergic disease. Curr Allergy Asthma Rep. 2011;11:421-6.

7. Liccardi G, Passalacqua G, Salzillo A, Piccolo A, Falagiani $P$, Russo M, Canonica GW, D'Amato $G$. Is sensitization to furry animals an independent allergic phenotype in non occupationally exposed individuals? J Investig Allergol Clin Immunol. 2011;21:137-41.

8. Liccardi G, Meriggi A, Russo M, Croce S, Salzillo A, Pignatti $P$. The risk of sensitization to furry animals in patients already sensitized to cat/dog: An in vitro evaluation using molecular-based allergy diagnostics. J Allergy Clin Immunol. 2015;135:1664-6.

9. Liccardi G, Piccolo A, Dente B, Salzillo A, Gilder JA, Russo M, D'Amato G. Rabbit allergens: a significant risk for allergic sensitization in subjects without occupational exposure. Respir Med. 2007;101:333-9.
10. Liccardi G, Bilò MB, Manzi F, Piccolo A, Di Maro E, Salzillo A. What could be the role of molecular-based allergy diagnostics in detecting the risk of developing allergic sensitization to furry animals? Eur Ann Allergy Clin Immunol. 2015;47:163-7.

Manuscript received June 15, 2016; accepted for publication July 20, 2016.

\section{Gennaro Liccardi}

Department of Pulmonology, Haematology and Oncology, Division of Pneumology and Allergology

High Speciality “A. Cardarelli” Hospital

Piazzetta Arenella 7

80128 Naples, Italy

E-mail: gennaro.liccardi@tin.it 